\title{
Coroa total de dente posterior em resina composta: relato de caso
}

Total crown of posterior tooth in composite resin: case report

Corona total de diente posterior con resina compuesta: reporte de caso

\author{
Bruno Firmino de OLIVEIRA ${ }^{1}$ \\ José Henrique de Araújo $\mathbf{C R U Z}^{2}$ \\ Douglas Benício Barros HENRIQUE ${ }^{1}$
}

Departamento de Pós-Graduação em Ortodontia, Instituto de Odontologia da Paraíba IOP, 58400-126 Campina Grande-PB, Brasil ${ }^{2}$ Curso de Graduação em Odontologia. Centro de Saúde e Tecnologia Rural, Universidade Federal de Campina Grande, UFCG 58708-110 Patos-PB, Brasil

\section{Resumo}

Introdução: Dentes que passaram por tratamento endodôntico e que perderam parte da estrutura dentária devido cárie, geralmente, necessitam de retentores intrarradiculares para promover retenção. Os padrões estéticos atuais, que buscam um sorriso de cor natural e alinhado, ocorreram nos últimos anos devido rápida evolução no que se diz respeito às resinas compostas. Objetivo: Reunir todas as vantagens desses biomateriais para reabilitar, harmonicamente, um pré-molar superior. Relato de Caso: Paciente procurou atendimento odontológico Odontominas para tratamento endodôntico do elemento 25 e após conclui-lo realizar reabilitação estética com pino de fibra de vidro e resina composta em consenso com o paciente após planejamento. Respeitando o princípio de desobturação de $2 / 3$ do canal radicular para inserção do pino com posterior reabilitação em resina composta, o pino foi cimentado com cimento resinoso Allcem dual na cor A2 FGM com auxílio do Lentulo $\mathrm{N}^{\circ} 35$, após ser preparado e adaptado ao conduto radicular. Posteriormente, o núcleo de preenchimento foi confeccionado e acabado com ponta diamantada $2135 \mathrm{~F}$ KG Sorense e condicionado com ácido fosfórico a $37 \%$ por 30 segundos, seguido do adesivo Single Bond $23 \mathrm{M} / \mathrm{ESPE}$ e foto ativado por 30 segundos. A subsequente reconstrução coronária foi realizada com pequenos incrementos fotopolimerizando cada camada, com sistema de resinas Z350 3M/ESPE para dentinana cor A3 e esmalte na cor A2. O pré-acabamento, foi realizado com ponta diamantada $2135 \mathrm{~F} \mathrm{KG}$ Sorensen e discos tipo sof-lex 3M/ESPE. O polimento da restauração foi feito após 48 horas, ponta de polimento Pogo Disco Dentsply e pasta própria para polimento.

Descritores: Materiais Dentários; Estética Dentária; Odontologia.

\section{Abstract}

Introduction: Teeth that have undergone endodontic treatment and which have lost part of the dental structure due to caries usually require intraradicular retainers to promote retention. The current esthetic standards, which seek a smile of natural color and aligned, have occurred in recent years due to rapid evolution in respect to composite resins. Objective: To combine all the advantages of these biomaterials to harmoniously rehabilitate a superior premolar. Case Report: Patient sought Odontominas dental care for endodontic treatment of element 25 and after completing it perform aesthetic rehabilitation with glass fiber pin and composite resin in consensus with the patient after planning. Respecting the disintegration principle of $2 / 3$ of the root canal for insertion of the pin with posterior restoration in composite resin, the pin was cemented with Allcem dual resin cement in A2 color FGM with the aid of Lentulo No. 35, after being prepared and adapted to the conduit root. Subsequently, the fill core was made and finished with 2135F KG Sorense diamond tip and conditioned with $37 \%$ phosphoric acid for 30 seconds, followed by Single Bond 2 3M / ESPE adhesive and activated photo for 30 seconds. The subsequent coronary reconstruction was performed with small increments by photopolymerizing each layer, with a 3M / ESPE resin system for dentinana A3 and enamel in color A2. The pre-finishing was performed with 2135F KG Sorensen diamond tip and 3M / ESPE sof-lex discs. The polishing of the restoration was done after 48 hours, polishing tip Pogo Disc Dentsply and own pulp for polishing.

Descriptors: Dental Materials; Esthetics, Dental; Dentistry.

\section{Resumen}

Introducción: Dientes que pasaron por tratamiento endodóntico y que perdieron parte de la estructura dental debido a la caries, por lo general, necesitan retenedores intrarradiculares para promover la retención. Los patrones estéticos actuales, que buscan una sonrisa de color natural y alineado, ocurrieron en los últimos años debido rápida evolución en lo que se refiere a las resinas compuestas. Objetivo: Reunir todas las ventajas de estos biomateriales para rehabilitar, armónicamente, un pre-molar superior. En la mayoría de los casos, la mayoría de las personas que sufren de depresión, que no se sienten satisfechas. Respetando el principio de desobturación de 2/3 del canal radicular para inserción del pino con posterior rehabilitación en resina compuesta, el pino fue cementado con cemento resinoso Allcem dual en el color A2 FGM con ayuda del Lenteulo $\mathrm{N}^{\circ} 35$, después de ser preparado y adaptado al conducto raíz. Posteriormente, el núcleo de relleno fue confeccionado y acabado con punta diamantada 2135F KG Sorense y acondicionado con ácido fosfórico a $37 \%$ por 30 segundos, seguido del adhesivo Single Bond $23 \mathrm{M} /$ ESPE y foto activado por 30 segundos. La posterior reconstrucción coronaria fue realizada con pequeños incrementos fotopolimerizando cada capa, con sistema de resinas Z350 3M / ESPE para dentinana color A3 y esmalte en el color A2. El preacabado, fue realizado con punta diamantada 2135F KG Sorensen y discos tipo sof-lex 3M / ESPE. El pulido de la restauración fue hecho después de 48 horas, punta de pulido Pogo Disco Dentsply y pasta propia para pulido.

Descriptores: Materiales Dentales; Estética Dental; Odontología.

\section{INTRODUÇÃO}

\section{Com o aumento da procura por} procedimentos estéticos por parte dos pacientes, verificou-se a necessidade de se realizar restaurações que se aproximam cada vez mais dos aspectos dos dentes naturais e que utilizem materiais que atendam aos requisitos físicos e mecânicos exigidos, tais como: biocompatibilidade, resistência às forças de mastigação, adaptação marginal, entre outros. Para a realização desses procedimentos, o profissional de odontologia tem ao seu dispor uma grande quantidade de materiais que possibilitam a execução de várias técnicas restauradoras ${ }^{1}$.

$$
\text { Por isso, graças às suas excelentes }
$$

propriedades estéticas e também as propriedades mecânicas e biológicas, as resinas compostas passaram a ser utilizadas também, nas restaurações de dentes posteriores, já que proporcionam um procedimento mais conservador, evitando desgaste dental e restringindo o preparo cavitário a remoção do tecido cariado ${ }^{2}$.

Para uma restauração ser considerada impecável, deve devolver estética e a função. Em algumas situações para que esses propósitos sejam alcançados, o uso de retentores intrarradiculares pode ser viável ${ }^{3}$. Quando a resistência e retenção à restauração no remanescente coronário não é 
satisfatória, pode haver necessidade de se conseguir a ancoragem dentro do canal radicular. Esta ancoragem pode ser obtida através de pinos pré-fabricados ${ }^{4}$.

Os pinos pré-fabricados, tais quais os pinos de fibra de vidro, oferecem praticidade e rapidez nos procedimentos de preparo do canal radicular e cimentação. Além de proporcionar preparo mais conservador, são de fácil manipulação, reduzem as sessões clínicas, potencializam adesividade, exibem propriedades biomecânicas próximas às do tecido dental e podem ser removidos facilmente do canal, em casos de necessidade de retratamento endodôntico ${ }^{5}$. Com base no exposto, objetivou-se reunir todas as vantagens desses biomateriais para reabilitar, esteticamente um pré-molar superior.

\section{CASO CLÍNICO}

Paciente do sexo masculino, leucoderma, 24 anos, foi encaminhado ao Departamento de Odontologia do Centro de Saúde Odontominas após realizar um tratamento endodôntico no elemento 25 para finalização do caso por meio da reabilitação estética e funcional do dente em questão.

Ao exame clínico, foi constatada total destruição coronária até o limite cervical, sem comprometimento periodontal. $\mathrm{O}$ elemento estava restaurado com material provisório (coltosol) (Figuras 1 e 2). Ao realizar análise radiográfica, foi verificado que o tratamento endodôntico estava satisfatório, apresentando $14 \mathrm{~mm}$ de material obturador em ambos os condutos radiculares (vestibular e palatino) e periápice saudável. Diante disso, e por questões individuais do paciente quanto ao planejamento e protocolo, optou-se por um tratamento reabilitador utilizando retentor intrarradicular estético (pino de fibra de vidro) associado à restauração direta de resina composta para reconstrução coronária.

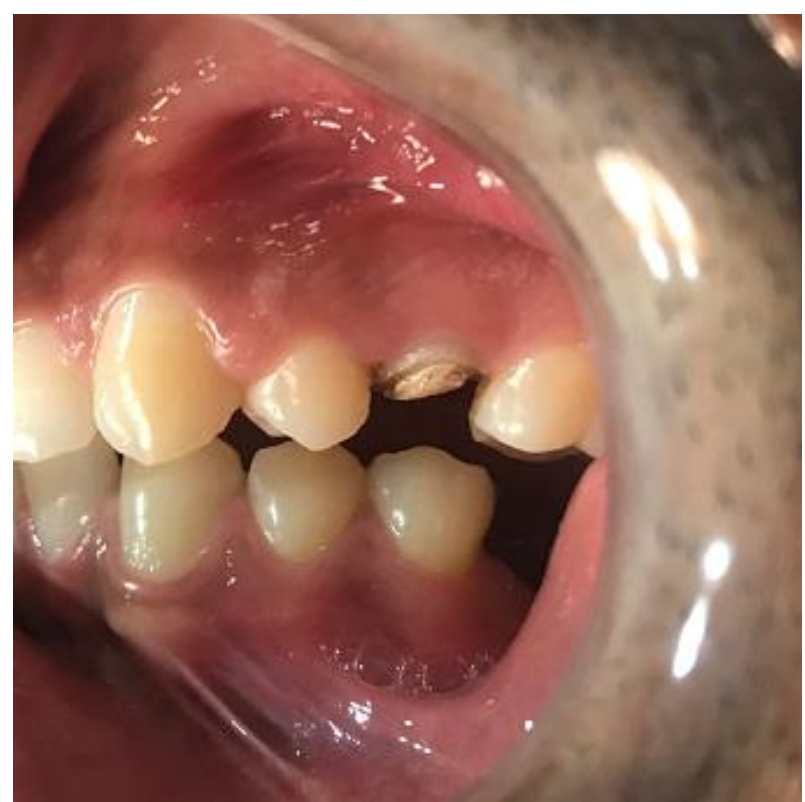

Figura 1: Aspecto clínico do elemento 25, vista vestibular

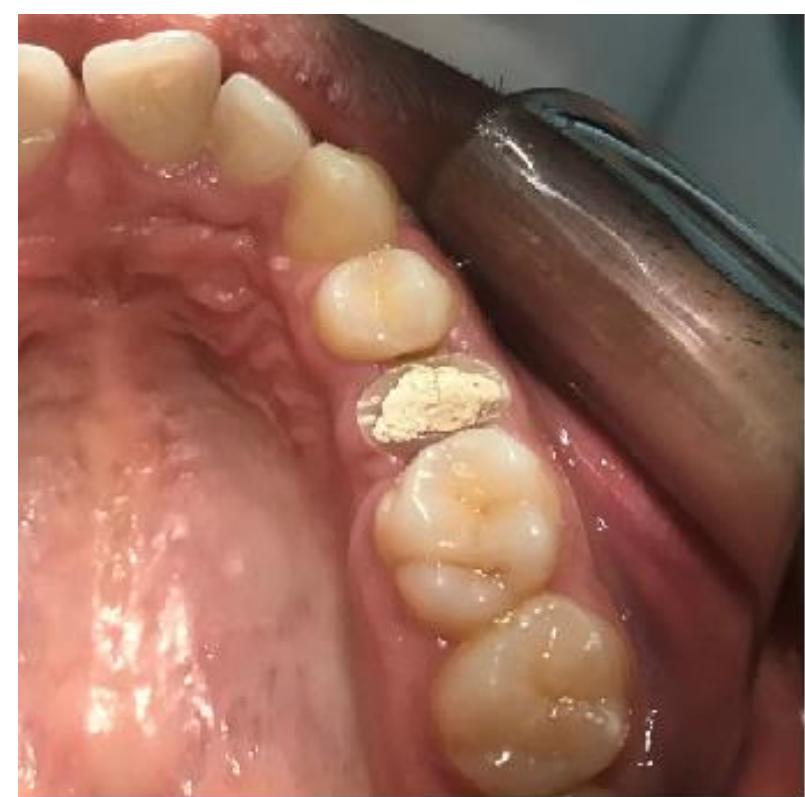

Figura 2: Aspecto clínico do elemento 25, vista oclusal.

Primeiramente, calculou-se a quantidade de material obturador a ser removido $(10 \mathrm{~mm})$, respeitando o protocolo de $4 \mathrm{~mm}$ de guta-percha para garantir o selamento apical. Iniciou-se a desobturação dos canais vestibular e palatino com brocas Largo $\mathrm{N}^{\circ}$ 2 e $\mathrm{N}^{\mathrm{o}} 1$ (Microdent ${ }^{\circledR}$, São Paulo, Brasil), confirmando-se o comprimento necessário $(10 \mathrm{~mm})$ com régua endodôntica milimetrada e cursor de borracha (Figura 3). Com auxílio de lima endodôntica tipo K-File \#20 de $25 \mathrm{~mm}$ (DentsplyMaileffer $^{\circledR}$, Rio de Janeiro, Brasil) e algodão embebido em álcool, os condutos foram limpos e secos com cones de papel absorvente.

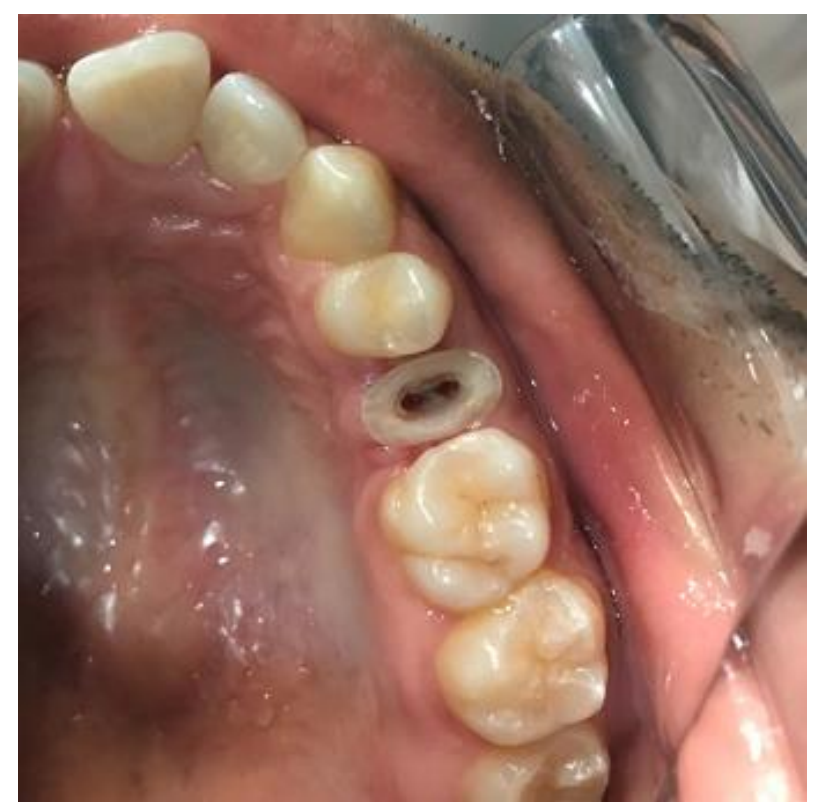

Figura 3: Desobturação dos condutos radiculares deixando $4 \mathrm{~mm}$ de guta percha.

Posteriormente, os retentores intrarradiculares em fibra de vidro foram provados nos condutos, sob isolamento relativo. Foi utilizado Reforpost $\mathrm{N}^{\circ} 1$ (Angelus ${ }^{\circledR}$, Londrina, Brasil) (Figuras 
4, 5 e 6). Para a cimentação do pino foi selecionado o cimento resinoso Allcem (FGM ${ }^{\circledR}$, São Paulo, Brasil) de presa dual e coloração A2, manipulado e inserido nos canais com auxílio de broca Lentulo $\mathrm{N}^{\circ} 35$ (Dentsply-Maileffer ${ }^{\circledR}$, Rio de Janeiro, Brasil) (Figura 7). Com os canais devidamente preenchidos, o cimento foi aplicado à superfície do pino previamente limpo com álcool e embebido em adesivo Single Bond 2 (3M/ESPE ${ }^{\circledR}$, São Paulo, Brasil) previamente fotoativado. Os pinos foram, então inseridos nos canais e o extravasamento de cimento retirado, seguido de fotopolimerização e do corte do excesso de pino em alta rotação com ponta diamantada $\mathrm{N}^{\circ}$ 2135F (KG Sorensen ${ }^{\circledR}$, São Paulo, SP, Brasil).

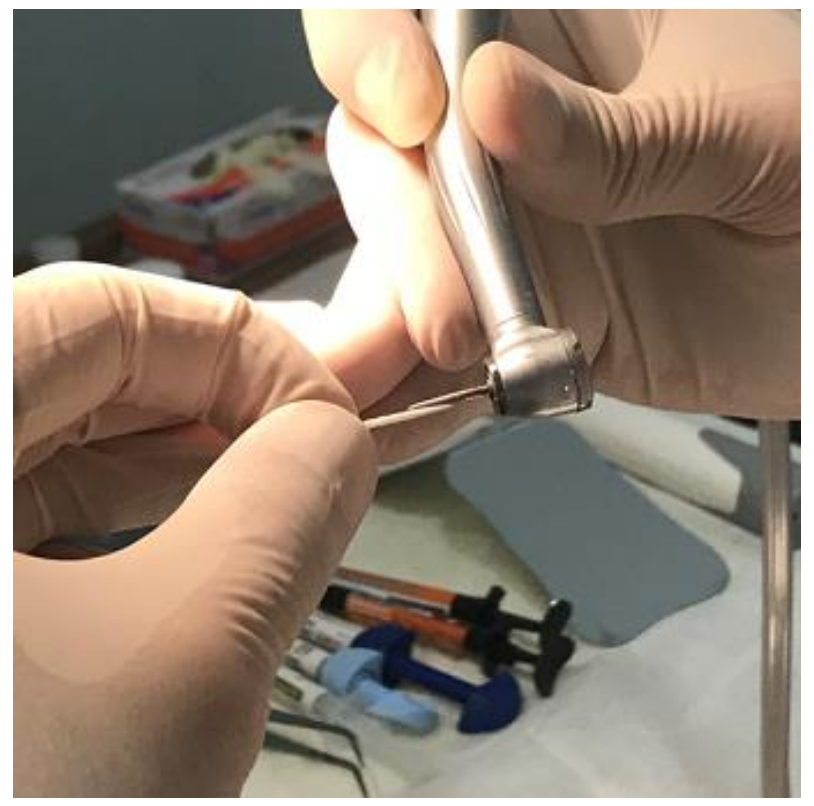

Figura 4: Prova e adaptação do pino de fibra de vidro aos condutos radiculares.

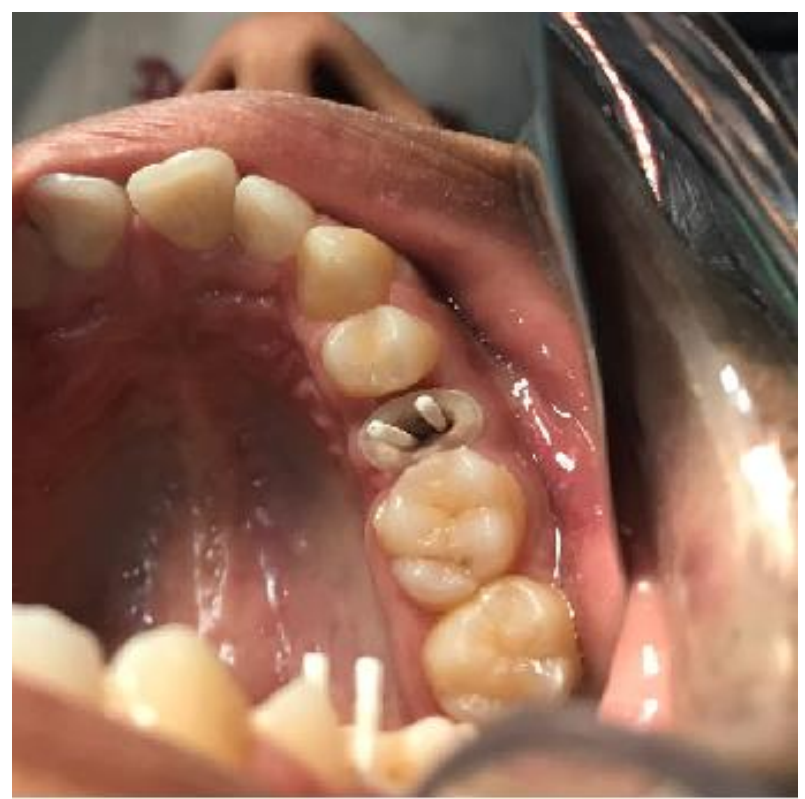

Figura 5: Prova e adaptação do pino de fibra de vidro aos condutos radiculares, vista oclusal.

Após cimentação dos retentores, seguiu-se o condicionamento da superfície dentária remanescente com ácido fosfórico a $37 \%$ (Biodinâmica ${ }^{\circledR}$, São Paulo, Brasil) por 30 segundos. Depois, foi feita a aplicação do adesivo Single Bond 2 (3M/ESPE ${ }^{\circledR}$, São Paulo, Brasil) com microbrush, seguido de fotopolimerização por 30 segundos.

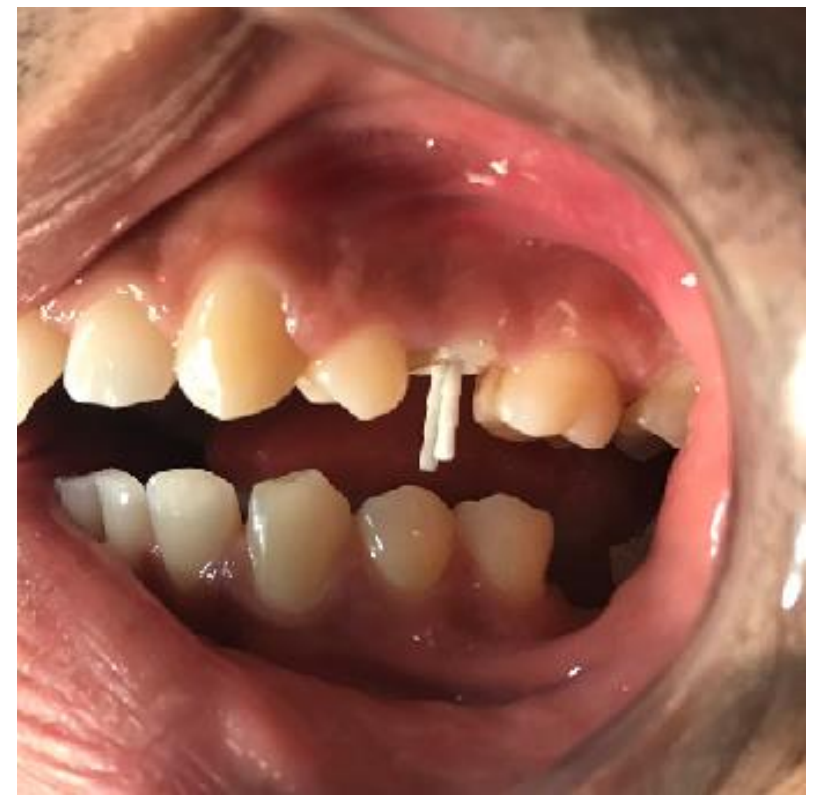

Figura 6: Prova e adaptação do pino de fibra de vidro aos condutos radiculares, vista vestibular.

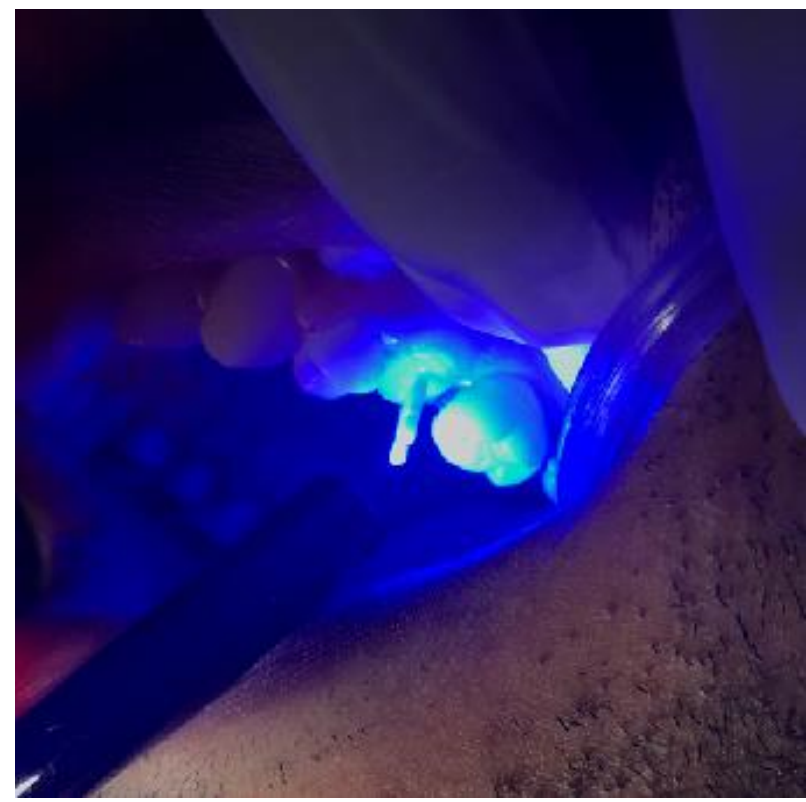

Figura 7: Fotoativação do cimento dual.

A reconstrução coronária foi realizada com o sistema de resinas Z350 (3M/ESPE ${ }^{\circledR}$, São Paulo, Brasil), sempre em pequenos incrementos, fotoativados individualmente, utilizando-se fotopolimerizador Optilight LD III (Gnatus ${ }^{\circledR}$, São Paulo, Brasil) com uma potência de $1200 \mathrm{~mW} / \mathrm{cm}$, para garantir um máximo de desempenho clínico do material. A face interna das cúspides recebeu incrementos de resina de corpo e dentina com cor A3 e a superfície foi produzida com compósitos para esmalte com coloração A3 e A2 (Figuras 8 e 9).

Em seguida foi realizado acabamento com 
ponta diamantada $N^{\circ} 2135 \mathrm{~F}$ (KG Sorensen ${ }^{\circledR}$, São Paulo, SP, Brasil) e discos tipo sof-lex (3M/ESPE ${ }^{\circledR}$, São Paulo, Brasil) em três granulações. $\mathrm{O}$ polimento da restauração foi feito após 48 horas, ponta de polimento Pogo Disco (Dentsply ${ }^{\circledR}$, Rio de Janeiro, Brasil) e pasta própria para polimento até a superfície apresentar-se perfeitamente lisa e brilhante.

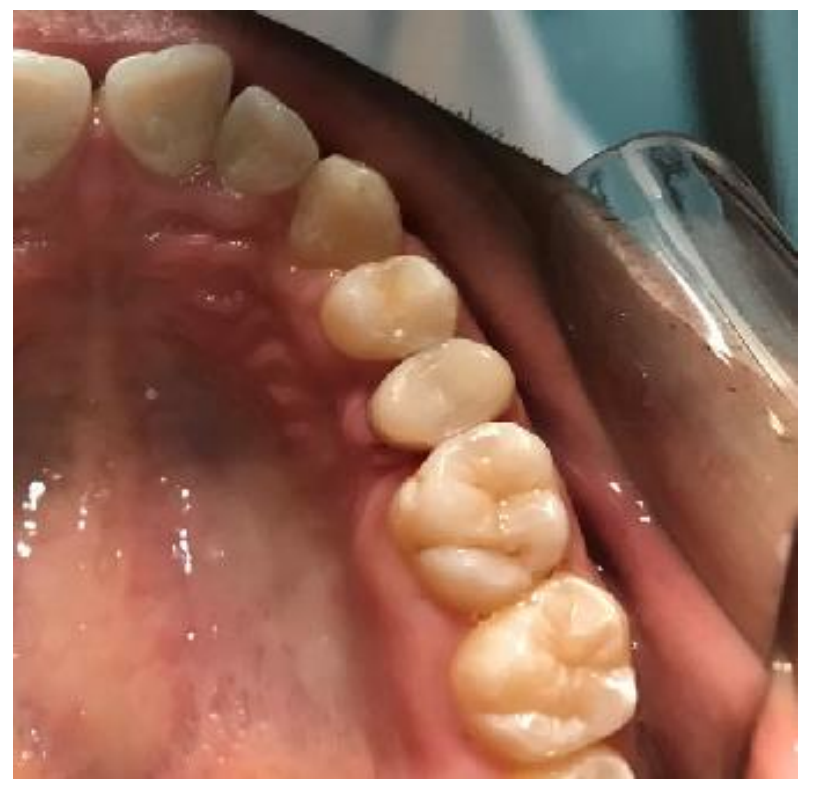

Figura 8: Reconstrução coronária com resina composta, vista oclusal.

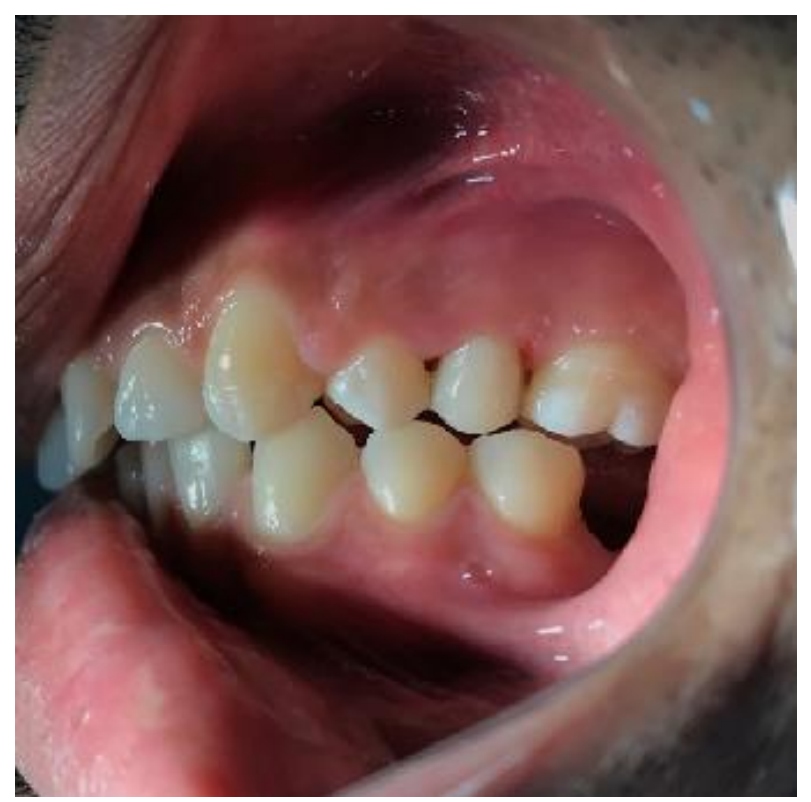

Figura 9: Reconstrução coronária com resina composta, vista vestibular.

\section{DISCUSSÃO}

A permanente busca por tratamentos odontológicos estéticos e por um sorriso elegante e harmônico é o que a maioria das pessoas deseja, o que colabora para a realização de procedimentos restauradores que possibilitem mimetização do natural para atingir as expectativas e necessidades dos pacientes ${ }^{6}$.

Para dentes que tiveram extensa perda de tecido dentário, o recurso mais indicado são os retentores intrarradiculares, e os pinos de fibra de vidro além de possibilitar seu efeito de recuperar a estrutura dentária perdida, produz resultado estético quando indicado ${ }^{7}$.

Algumas características anatômicas dos elementos dentários devem ser analisadas antes da inserção dos pinos de vibra de vidro, bem como: morfologia e comprimento radicular, localização do elemento na arcada dentário, tipo de oclusão do paciente, opções de material restaurador, e a expectativa da longevidade da restauração e da estética do paciente ${ }^{8,9}$.

Posto isso, uma vez que a dentina é reconstruída por pino de fibra de vidro e resina composta, os princípios de biomimetismo de estrutura dental são conseguidos na sua plenitude, materiais que apresentam módulo de elasticidade, e propriedades mecânicas semelhantes à dentina ${ }^{7}$.

Além disso, a união adesiva nas paredes dos canais por meio do protocolo adesivo utilizado se torna possível devido às características físicas dos pinos permitirem esta função. Logo, formando um corpo único em virtude da cimentação adesiva, o que promove a melhor distribuição de tensões no interior do canal a ao remenescente radicular ${ }^{10}$.

A associação entre fotoativação e polimerização química dos cimentos duais, devido sua composição, proporcionam propriedades físicas e mecânicas, como força de união, resistência ao desgaste e resistência à compressão superior aos demais materiais de cimentantes, todavia, o maior inconveniente é controle da umidade durante a cimentação ${ }^{11}$.

A composição dos materiais restauradores resinosos tem passado por diversas mudanças ao longo dos últimos 50 anos, em especial no que diz respeito ao aumento da carga inorgânica de dimensões reduzidas, em busca de se obter uma maior estética de polimento sem comprometimento da resistência mecânica da restauração. Atualmente, os objetivos a serem atingidos visam numa menor contração de polimerização e uma possível autoadesão do material ao substrato ${ }^{12}$.

Os materiais de restauração à base de resina apresentam diferenças em suas composições, no entanto a sua semelhança reside nos quatro componentes estruturais básicos: uma matriz polimérica, partículas de carga inorgânica (geralmente vidros de sílica radiopacos), silano e promotores/reguladores da reação de polimerização ${ }^{12}$.

As resinas compostas fotopolimerizáveis são um importante grupo de materiais restauradores da Odontologia, pois podem ser usadas para restaurar dentes e assim devolver forma e função aos elementos, apresentam propriedades mecânicas justificáveis, além de polimerização imediata após fotopolimerização ${ }^{13}$.

A contração de polimerização ainda é 
considerada uma carência para as restaurações de resina composta. Nesse sentido, Park et al. ${ }^{13}$ desenvolveram um estudo que objetivou avaliar o grau de polimerização de compósitos de resina de baixa contração de polimerização e suas propriedades de flexão, compressão e contração, utilizando cinco compósitos de resina a base de metacrilato (Aelite LS Posterial; Grandio; Premise; Estelite Sigma Quick e Venus Diamond) e um a base de silorano (Filtek LS), curados com três unidades diferentes de polimerização à luz (quartzo-tungstênio-halogênio e dois emissores de luz (LEDs) previamente verificada a intensidade da luz de cada unidade com radiômetro, (aproximadamente $900 \mathrm{~mW} / \mathrm{cm}^{2}$ ). Com base nos resultados, os autores observaram que: a escolha da luz de polimerização não tem relação específica com as propriedades das resinas; a força de flexão não teve relação com a unidade de luz utilizada nem com o conteúdo de enchimento. Entre os compósitos avaliados, o Filtek apresentou força de flexão de media a alta e força de compressão mais baixa. Relacionaram a contração de polimerização com o volume do conteúdo de resina e mostraram que a Filtek apresentou a menor contração de polimerização em relação aos outros compósitos testados. Segundo os autores, tanto o módulo de flexão, quanto o módulo compressivo mantêm correlação com o conteúdo de enchimento (compósito de resina) e essas propriedades dos espécimes foram semelhantes a dentina subjacente.

Schneider et al. ${ }^{14}$ demostraram através de estudo a influência de três diferentes modos de fotopolimerização - Convencional (CONV), Softstart (SS) e Pulso atrasado (PA) - sobre a microdureza de três resinas compostas - Filtek Z350 (3M ESPE), Empress Direct (IvoclarVivadent) e P90 (3M ESPE). Obtiveram nove grupos para os experimentos e realizaram o teste de microdureza Knoop nos corpos de prova 24 horas após a polimerização. Com a análise dos testes, os autores concluíram que os diferentes modos de fotopolimerização não interferem na eficácia da polimerização das resinas compostas testadas. Enfatizaram ainda que, dentre as resinas testadas, a Empress Direct foi a que apresentou os valores mais baixos de dureza para os três modos de polimerização.

Comportamento clínico satisfatório é verificado nas restaurações com resina, porém neste procedimento é necessário maior desgaste da estrutura dentária para o preparo da cavidade e do conducto radicular ${ }^{15}$. Para Cruz et al. ${ }^{16}$ o desempenho profissional mais consciente sobre a anatomia e habilidade manual, recuperando forma e função, fisiologia mastigatória, harmonia e estética entre os arcos, é de fundamental importância para reconstrução coronária de um dente com extensa perda.

\section{CONCLUSÃO}

Portanto, junto ao fato das inúmeras pesquisas terem melhorado significativamente as resinas compostas e devido às restaurações com esse tipo de material representar uma das atividades mais desenvolvidas na Odontologia moderna, as restaurações de reconstrução coronárias em resina composta são, hoje, uma alternativa às coroas fixas protéticas para recuperação da estética e função.

\section{REFERÊNCIAS}

1. Behr M, Proff P, Kolbeck C, Langriegr S, Kunze $\mathrm{J}$, Handel $\mathrm{G}$ et al. The bond strength of the resinto-zirconia interface using diferente bonding concepts. J Mech Behav Biomed Mater. 2011; 4(1):2-8.

2. Rodrigues DS. Análise da porosidade, resistência mecânica e desgaste de cimentos de ionômero de vidro e resina composta [dissertação]. Porto (Portugal): Faculdade de Ciências da Saúde, Universidade Fernando Pessoa; 2014.

3. Della-Bona A. Bonding to ceramics: scientific evidences for clinical dentistry. São Paulo: Artes Médicas; 2009.

4. Assunção DPO. Pinos intrarradiculares préfabricados de fibra de vidro. Piracicaba: Faculdade de Odontologia de Piracicaba UNICAMP; 2011. Trabalho de Conclusão de Curso.

5. Zogheib LV. Resistência à fratura de raízes fragilizadas restauradas com resina composta e pinos de fibra de vidro submetidas à ciclagem mecânica [dissertação]. Bauru: Faculdade de Odontologia USP; 2005.

6. Barcellos CR, Silva FP, Carneiro RP, Borges MG, Pereira DA, Menezes MS. Reabilitação funcional e estética do sorriso: um relato de caso. Full dent. Sci. 2015;7(25):102-13.

7. Alfredo E, de Souza ES, Marchesan MA, Paulino SM, Gariba-Silva R, Sousa-Neto MD. Effect of eugenol-based endodontic cement on the adheston of intra-canal posts. Braz Dent J. 2006;17(2):130-33.

8. Reis BR, Soares PBF, Castro CG, Santos Filho PCF, Soares PV, Soares CJ. Uso de coroa em cerâmica pura associada a pino de fibra de vidro na reabilitação estética do sorriso: relato de caso. Rev Odontol Bras Central. 2010;19(50):262-69.

9. Muniz L. Reabilitação estética em dentes tratados endodonticamente: pinos de fibra e possibilidades clínicas conservadores. São Paulo: Santos; 2010.

10.Menezes MS, Queiroz EC, Campos RE, Martins LR, Soares CJ. Influence of endodontic sealer cement on berglass post bond strength to root dentine. Int Endod J. 2008;41(6):476-84.

11.Prakki A, Carvalho RM. Dual cure resin cements: characteristics ans clinical considerations. PósGrad Rev Fac Odontol São José dos Campos. 2001;4(1):21-6. 
12.Ferracane JL. Resin composite - state of the art. Dent Mater. 2011; 27(1):29-38.

13.Park JK, Lee GH, Kim JH, Park MG, Ko CC, Kim $\mathrm{H}$ et al. Polymerization shrinkage, flexural and compression properties of low-shrinkage dental resin composites. Dent Mater J. 2014; 33(1):104-10.

14. Schneider AC, Mendonça MJ, Rodrigues RB, Busato PMR, Camilotti V. Influência de três modos de fotopolimerização sobre a microdureza de três resinas compostas. Polímeros. 2016;26(Esp):37-42.

15. Sousa LX, Cruz JHA, Melo WOS, Freire SCP, Ribeiro ED, Freire JCP. Abfração dentária: um enfoque sobre a etiologia e o tratamento restaurador. Arch Health Invest. 2018;7(2):51-3.

16.Cruz JHA, Silva RLB, Andrade-Júnior FP, Guênes GMT, Almeida MSC, Medeiros LADM et al. A importância da anatomia e escultura dental para prática de procedimentos clínicos odontológicos. RSC. 2018;7(1):76-85.
CONFLITO DE INTERESSES

Os autores declaram não haver conflitos de interesse.

AUTOR PARA CORRESPONDENCIA

\section{Bruno Firmino de Oliveira}

bruno_firmino19@hotmail.com

Submetido em 09/08/2018

Aceito em 04/10/2018 